\title{
Shape Priors using Manifold Learning Techniques
}

\author{
Patrick Etyngier Florent Ségonne Renaud Keriven \\ Odyssée Project, CERTIS, Ecole des ponts / INRIA / ENS \\ \{etyngier, segonne, keriven\}@certis.enpc.fr
}

\begin{abstract}
We introduce a non-linear shape prior for the deformable model framework that we learn from a set of shape samples using recent manifold learning techniques. We model a category of shapes as a finite dimensional manifold which we approximate using Diffusion maps, that we call the shape prior manifold. Our method computes a Delaunay triangulation of the reduced space, considered as Euclidean, and uses the resulting space partition to identify the closest neighbors of any given shape based on its Nyström extension.

Our contribution lies in three aspects. First, we propose a solution to the pre-image problem and define the projection of a shape onto the manifold. Based on closest neighbors for the Diffusion distance, we then describe a variational framework for manifold denoising. Finally, we introduce a shape prior term for the deformable framework through a non-linear energy term designed to attract a shape towards the manifold at given constant embedding. Results on shapes of cars and ventricule nuclei are presented and demonstrate the potentials of our method.
\end{abstract}

\section{Introduction}

\subsection{Motivation}

Image segmentation is an ill-posed problem due to various perturbing factors such as noise, occlusions, missing parts, cluttered data, etc. When dealing with complex images, some prior shape knowledge may be necessary to disambiguate the segmentation process. The use of such prior information in the deformable model framework has long been limited to a smoothness assumption or to simple parametric families of shapes. But a recent and important trend in this domain is the development of deformable models integrating more elaborate shape information.

An important work in this direction is the active shape model of Cootes et al. [8]. A principal component analysis (PCA) on the position of some landmark points placed in a coherent way on all the training contours is used to reduce the number of degrees of freedom to the principal modes of variation. Although successfully applied to various types of shapes (hands, faces, organs), the reliance on a parameterized representation and the manual positioning of the landmarks, particularly tedious in 3D images, seriously limits it applicability.

Leventon, Grimson and Faugeras [17] circumvent these limitations by computing parameterization-independent shape statistics within the level set representation [20]. Basically, they perform a PCA on the signed distance functions of the training shapes, and the resulting statistical model is integrated into a geodesic active contour framework.The evolution equation contains a term which attracts the model toward an optimal prior shape as a combination of the mean shape and of the principal modes of variation. Several improvements to this approach have been proposed [21, 23], and in particular an elegant integration of the statistical shape model into a unique MAP Bayesian optimization. Let us also mention another neat Bayesian prior shape formulation, based on a B-spline representation, proposed by Cremers, Kohlberger and Schnörr in [9].

Performing PCA on distance functions might be problematic since they do not define a vector space. To cope with this, Charpiat, Faugeras and Keriven [5] proposed shape statistics based on differentiable approximations of the Hausdorff distance. However, their work is limited to a linearized shape space with small deformation modes around a mean shape. Such an approach is relevant only when the learning set is composed of very similar shapes.

Lastly, note that the method presented in this paper is different and far superior to the preliminary work introduced in [11].

\subsection{Novelty of our Approach}

In this paper, we depart from the small deformation assumption and introduce a new deformable model framework that integrates more general non-linear shape priors. We model a category of shapes as a smooth finitedimensional sub-manifold of the infinite-dimensional shape space, termed the shape prior manifold. This manifold which cannot be represented explicitly is approximated 
from a collection of shape samples using a recent manifold learning technique called diffusion maps [7, 16]. Manifold learning, which is already an established tool in object recognition and image classification, has been recently applied to shape analysis [6]. Yet, to our knowledge, such techniques have not been used in the context of image segmentation with shape priors.

Diffusion maps generate a mapping, called an embedding, from the original shape space into a low-dimensional space. Advantageously, this mapping is an isometry from the original shape space, equipped with a diffusion distance, into a low-dimensional Euclidean space [7]. In this paper, we exploit the isometrical mapping and the Euclidean nature of the reduced space to design our variational shape prior framework. We propose to introduce a shape prior term for the deformable framework through a non-linear energy term designed to attract a shape towards its projection onto the manifold. Doing so requires being able to estimate the manifold between training samples and to compute the projection of a shape onto the manifold. Unfortunately, diffusion maps do not give access to such tools.

Our contribution lies in three aspects. First, we propose a solution to the estimation of the manifold between training samples. We define a projection operator onto the manifold based on: 1) Nyström extensions [3] which provide a sound and efficient framework for extending embedding coordinates (in the shape prior manifold) to the full infinite dimensional shape space, 2) a Delaunay partitioning of the reduced space to identify the closest neighbors (in the training set) of any shape in the original infinite dimensional shape space. In light of this, we then describe a variational framework for manifold denoising, thereby lessening the negative impact of outliers onto our variational shape framework. Finally, we describe our shape prior term integrated in the deformable model framework through a nonlinear energy term designed to attract a shape towards the manifold at given constant embedding.

The remainder of this paper is organized as follows. Section 2 introduces the necessary background in manifold learning: it is dedicated to learning the shape prior manifold from a finite set of shape samples using diffusion maps. Section 3 describes our contributions. Section 4 reports some preliminary numerical experiments which yield promising results with real shapes.

\section{Learning the Shape Prior Manifold}

In the sequel, we define a shape as a simple compact (i.e. bounded closed and non-intersecting) surface, and $\mathbb{S}$ denotes the (infinite-dimensional) space of such shapes. Note that, although this paper only deals with 2-dimensional surfaces embedded in the 3-dimensional Euclidean space, all ideas and results seamlessly extend to higher dimensions. We make the assumption that a category of shapes can be modeled as a finite-dimensional manifold (the shape prior manifold)

Dimensionality reduction, i.e. the process of recovering the underlying low dimensional structure of a manifold that is embedded in a higher-dimensional space, has seen renewed interest over the past years. Among the most recent and popular techniques are the Generalized Multi-Dimensional Scaling [4], the Locally Linear Embedding (LLE) [22], Laplacian eigenmaps [2], diffusion maps [7, 15]. Most of these techniques construct an adjacency graph of the learning set of shape samples and map the data points into a lower dimensional space while preserving the local properties of the adjacency graph. This dimensionality reduction with minimal local distortion can be achieved using spectral methods, i.e. through an analysis of the eigen-structure of some matrices derived from the adjacency graph.

In this work, we learn the shape prior manifold using diffusion maps, since their extension to infinite-dimensional (shape) manifolds is straightforward (see [7, 16, 15] for more details).

\subsection{Diffusion Maps}

Let $\mathcal{M}$ be a manifold of dimension $m$ lying in $\mathbb{S}$. Diffusion maps rely on discrete approximations of the LaplaceBeltrami operator $\Delta_{\mathcal{M}}$ defined on the manifold $\mathcal{M}$ to generate a mapping (called an embedding) $f: \mathcal{M} \longrightarrow \mathbb{R}^{m}$ such that if two points $x$ and $z$ are close in $\mathcal{M}$, then also are $f(x)$ and $f(z)$. The optimal mapping is given by the eigenvalues and eigenfunctions of the Laplace-Beltrami operator corresponding to the $m$ smallest non-zero eigenvalues, where $m$ is the target dimension. Note that the latter dimension can either be known a priori or inferred from the profile of the eigenspectrum [7].

In practice, a discrete counterpart to this continuous formulation must be used since we only have access to a discrete and finite set of example shapes in a given category. We will assume that this set constitutes a "good" sampling of the shape prior manifold, where "good" stands for "exhaustive" and "sufficiently dense" in a sense that will be clarified below [12].

\subsubsection{Distance in the Shape Space}

The approximation of the Laplace-Beltrami operator requires the choice of a distance between shapes. Many different definitions of the distance between two shapes have been proposed in the computer vision literature but there is no agreement on the correct way to measure shape similarity. We represent a surface $s$ in the Euclidean embedding space $\mathbb{R}^{3}$ by its signed distance function $\bar{D}_{s}$. In this context, we define the distance between two shapes to be the Sobolev $W^{1,2}$-norm of the difference between their signed 
distance functions [5]:

$$
\begin{aligned}
d_{W^{1,2}}\left(s_{1}, s_{2}\right)^{2}= & \left\|\bar{D}_{s_{1}}-\bar{D}_{s_{2}}\right\|_{L^{2}(\Omega, \mathbb{R})}^{2} \\
& +\left\|\nabla \bar{D}_{s_{1}}-\nabla \bar{D}_{s_{2}}\right\|_{L^{2}\left(\Omega, \mathbb{R}^{n}\right)}^{2},
\end{aligned}
$$

where $\bar{D}_{s_{i}}$ denotes the signed distance function of shape $s_{i}$ $(i=1,2)$, and $\nabla \bar{D}_{s_{i}}$ its gradient. Note that to define a distance between shapes that is invariant to rigid displacements (e.g. rotations and translations), we first align the shapes using their principal moments before computing distances. Note also that the proposed method is obviously not limited to a specific choice of distance [5].

\subsubsection{Discrete Laplace-Beltrami Operator}

Once a distance has been chosen, classical manifold learning techniques can be applied by building an adjacency graph of the learning set of shape examples. Let $\Gamma=$ $\left\{s_{1} \cdots s_{p} \in \mathbb{S}\right\}$ be $p$ sample points of the $m$ dimensional manifold $\mathcal{M}$ sampled under an unknown density $q_{\mathcal{M}}$ $(m \leq p)$. An adjacency matrix $\left(W_{i, j}\right)_{i, j \in 1, \ldots, p}$ is then constructed, the coefficients of which measure the strength of the different edges in the adjacency graph. Typically, $W_{i, j}$, also denoted $w\left(s_{i}, s_{j}\right)$, is a decreasing function of the distance between shapes $s_{i}$ and $s_{j}$. In this work, we use the Gaussian kernel $w\left(s_{i}, s_{j}\right)=\exp \left(-d_{W^{1,2}}^{2}\left(s_{i}, s_{j}\right) / 2 \sigma^{2}\right)$, with $\sigma$ estimated as the median of all the distances between all shapes [15].

Classical manifold learning methods provide an embedding that combines the information of both the density $q_{\mathcal{M}}$ and the geometry $[12,15,16]$. In order to construct an approximation of the Laplace-Beltrami operator that is independent of the unknown density $q_{\mathcal{M}}$, we renormalize the adjacency matrix $\left(W_{i, j}\right)$. Briefly, we form the new adjacency matrix $\left(\tilde{W}_{i, j}\right)$ by $\tilde{w}\left(s_{i}, s_{j}\right)=\frac{w\left(s_{i}, s_{j}\right)}{q\left(s_{i}\right) q\left(s_{j}\right)}$, with $q(s)=$ $\sum_{y \in \Gamma} w(s, y)$ being the Nadaraya-Watson estimate of the density $q_{\mathcal{M}}$ at location $s$ (up to a normalization factor). We then define the anisotropic transition kernel $\left(P_{i, j}\right)_{i, j \in 1, \ldots, p}$ such that $p\left(s_{i}, s_{j}\right)=\frac{\tilde{w}\left(s_{i}, s_{j}\right)}{\tilde{q}\left(s_{i}\right)}$ with $\tilde{q}(s)=\sum_{y \in \Gamma} \tilde{w}(s, y)$. From the definition of the adjacency matrix, we find that:

$$
p\left(s_{i}, s_{j}\right)=\frac{w\left(s_{i}, s_{j}\right)}{\sum_{b \in \Gamma} K_{b}^{j} w\left(s_{i}, s_{b}\right)} \text { with } K_{b}^{j}=\frac{q\left(s_{j}\right)}{q\left(s_{b}\right)} .
$$

The kernel $\left(\mathbb{1}-P_{i, j}\right)$ is then a density-independent approximation of the Laplace-Beltrami operator $\Delta_{\mathcal{M}}[7,12]$.

\subsubsection{Generating the Embedding using diffusion Maps}

Let $\left(\lambda_{i}\right)_{i \in 1, \ldots, p}$ with $\lambda_{0}=1 \geq \lambda_{1} \geq \cdots \geq 0$ and $\left(\Psi_{i}\right)_{i \in 1, \ldots, p}$ be respectively the eigenvalues and the associated eigenvectors of $\left(P_{i, j}\right)$. Coifman and coworkers have shown in [7] that the eigenvectors of $\left(P_{i, j}\right)$ converge to those of the Laplace-Beltrami operator on $\mathcal{M}$ and that a mapping $\Phi$ that embeds the data into the Euclidean space $\mathbb{R}^{m}$ quasi-isometrically ${ }^{1}$ with respect to a diffusion distance in the original shape space $\mathbb{S}$ can be constructed as:

$$
\Phi: \Gamma \subset \mathcal{M} \rightarrow \mathbb{R}^{m}, s_{i} \mapsto\left(\lambda_{1}^{\rho} \Psi_{1}\left(s_{i}\right), \ldots, \lambda_{m}^{\rho} \Psi_{m}\left(s_{i}\right)\right)
$$

Diffusion distance reflects the intrinsic geometry of the data set defined via the adjacency graph in a diffusion process (the anisotropic kernel $\left(P_{i, j}\right)$ being seen as a transition matrix in a random walk process). In this formulation, $\rho$ is a parameter controlling the diffusivity of the adjacency graph and can be chosen arbitrarily. We used $\rho=1$ for our experiments. Diffusion distance was shown to be more robust to outliers than geodesic distances [7], thereby motivating its use to estimate the embedding (Fig.). Accordingly, in the remainder of this paper, the notion of proximity in the original shape space (e.g. the "closest" neighbors of a given shape) is based on the diffusion distance. Since the embed$\operatorname{ding} \Phi$ is an isometry, proximity is advantageously deduced in the Euclidean reduced space.

\subsection{Nyström Extensions}

The mapping $\Phi$ is only defined on the training samples. The Nyström extension method is a popular technique employed for the extension of empirical functions from the training set $\Gamma$ to new samples, i.e. the out of sample problem $[3,1]$. Noticing that every training sample verifies:

$\forall x \in \Gamma \quad \forall k \in 1, \ldots, p \quad \sum_{y \in \Gamma} p(x, y) \Psi_{k}(y)=\lambda_{k} \Psi_{k}(x)$,

the embedding of new data points located outside the set $\Gamma$ can similarly be computed by a smooth extension $\tilde{\Phi}$ of $\Phi$ (Lafon and coworkers define another elegant extension in [15]):

$\tilde{\Phi}:\left\{\begin{array}{c}\mathbb{S} \rightarrow \mathbb{R}^{m}, s \mapsto\left(\tilde{\Phi}_{1}(s), \ldots, \tilde{\Phi}_{m}(s)\right) \\ \forall k \in 1, \ldots, p \tilde{\Phi}_{k}(s)=\lambda_{k}^{\rho-1} \sum_{y \in \Gamma} p(s, y) \Psi_{k}(y)\end{array}\right.$

\section{Image Segmentation using the Shape Prior Manifold}

In this section, we propose to use the embedding to carefully design a shape prior term integrated into a deformable model framework for the purpose of image segmentation. Without loss of generality, we cast the segmentation problem as a variational one, where the objective is to find a surface $S$ minimizing a global energy functional $E^{a c}$. Depending on the segmentation task and the available information, the energy functional $E^{a c}$ can take on different, more or less complex, forms, but, generally, $E^{a c}$ can be written as

\footnotetext{
${ }^{1}$ it is an isometry when $m=p$
} 
a combination of image terms, designed to drive the surface toward the searched contour, and regularization terms, enforcing smoothness constraints. Directly finding the global minimum of $E^{a c}$ is usually impossible and one often has to resort to a sub-optimal gradient-descent strategy starting from a guess $S_{0}$. That is we assume that the image segmentation problem amounts to solving the following evolution problem: find the active contour $s: \tau \in \mathbb{R}^{+} \mapsto s(\tau) \in \mathbb{S}$ such that $s(0)=s_{0}, \frac{d s}{d \tau}=-\nabla E^{a c}$.

We introduce into the evolution equation a shape prior term designed to attract a given shape $s \in \mathbb{S}$ towards its projection onto the manifold, denoted $p_{\mathcal{M}}(s)$. Unfortunately, diffusion maps do not permit the explicit estimation of the manifold between training samples (i.e. the pre-image problem) and do not give access to an explicit projection operator onto the manifold $\mathcal{M}$. In addition, training samples might include outliers that might deteriorate the behavior of our shape prior.

Therefore, we propose to design our shape prior in three steps, which constitute our three main contributions. First, we propose a solution to the estimation of the manifold between training samples and define a projection operator onto the manifold. We then introduce a manifold denoising framework for the purpose of lessening the negative impact of outliers. Finally, we describe our shape prior term integrated in the deformable model framework through a nonlinear energy term designed to attract a shape towards the manifold at given constant embedding.

We will use the following notations. We denote $\mathbb{S}_{x}=$ $\tilde{\Phi}^{-1}(x)$ is the $x$-level set in $\mathbb{S}$ of the embedding $\tilde{\Phi}$. Note that $\left\{\mathbb{S}_{x}, x \in \mathbb{R}^{m}\right\}$ realizes a partition of $\mathbb{S}$ into sub-manifolds of codimension $m . \tilde{\Phi}_{\mid \mathcal{M}}^{-1}(x)$ denotes the shape $s$ in $\mathcal{M}$ whose embedding coordinates are $\tilde{\Phi}(s)=x$, i.e. $\tilde{\Phi}_{\mid \mathcal{M}}^{-1}(x)=\mathcal{M} \cap$ $\mathbb{S}_{x}$. Finally, note also that the projection of any shape $s$ onto the manifold is $P_{\mathcal{M}}(s)=\tilde{\Phi}_{\mid \mathcal{M}}^{-1}(\tilde{\Phi}(s))$.

\subsection{Manifold Estimation and Projection}

Given a point in the reduced space $x \in \mathbb{R}^{m}$, we endeavor to find the shape $s=\tilde{\Phi}_{\mid \mathcal{M}}^{-1}(x)$ in the manifold $\mathcal{M}$ such that $\tilde{\Phi}(s)=x$, i.e. the pre-image of $x$ [14]. As noted by Arias and coworkers in [1], such a shape $S$ might not exist and the pre-image problem is ill-posed. To circumvent this problem, they search for a pre-image that optimizes a given optimality criterion in the reduced space.

In this work, we take a different approach. We are only interested in estimating the manifold $\mathcal{M}$ between "neighboring" training samples. Therefore, we assume that the point $x \in \mathbb{R}^{m}$ falls inside the convex-hull of the training samples in the reduced space (were the point $x \in \mathbb{R}^{m}$ outside, we would consider instead its orthogonal projection on the convex-hull). In this sense, the set of training samples must be exhaustive enough to capture the limits of the manifold $\mathcal{M}$.

We also assume that the shape $s$, belonging to the manifold $\mathcal{M}$, can be expressed as a weighted mean shape [5] that interpolates between "neighboring" samples for the diffusion distance. To this end, we exploit the Euclidean nature of the reduced space $\mathbb{R}^{m}$ to determine the $m+1$ closest neighbors of $s$ (note that if the point $x \in \mathbb{R}^{m}$ is located outside the convex-hull, then only $m$ neighbors are identified.). In this sense, the set of training samples must be sufficiently dense for the interpolation to be meaningful.

We compute a Delaunay triangulation $\mathbb{D}_{\mathcal{M}}$ in the reduced space of the training data and identify the $m+1$ closest neighbors of $s$ as the $m+1$-Delaunay triangle that $x$ belongs to. This $m$-dimensional triangle is formed by $m+1$ points that correspond to the image by $\tilde{\Phi}$ of the $m+1$ closest neighbors $\mathcal{N}=\left(s_{0}, \ldots, s_{m}\right)$ of $s$ in $\mathbb{S}$ for the diffusion metric [7].

Having identified the $m+1$ closest neighbors $\mathcal{N}=$ $\left(s_{0}, \ldots, s_{m}\right)$ of $s$, we define the pre-image image of $x$ as the solution to the optimization problem:

$$
s=\arg \min _{\theta_{i}, s} \sum_{s_{i} \in \mathcal{N}} \theta_{i} d^{2}\left(s, s_{i}\right) \text { such that } \tilde{\Phi}(s)=x,
$$

with $\left(\theta_{i} \geq 0, \sum_{i=0}^{m} \theta_{i}=1\right)$. The coefficients $\Theta=$ $\left\{\theta_{0}, \ldots, \theta_{m}\right\}$ are the barycentric coefficients of the the shape $s$ with respect to its neighbors $\mathcal{N}$ in the shape space $\mathbb{S}$ equipped with the diffusion distance. In practice, the preimage $\tilde{\Phi}_{\mid \mathcal{M}}^{-1}(x)$ and the associated coefficients $\Theta$ are computed by gradient descent, with an initial guess provided by the barycentric coordinates of the image $x$ in the reduced space: $x=\sum \theta_{i} \tilde{\Phi}\left(s_{i}\right)$. Figure 1 illustrates our projection operator on a 2 dimensional manifold lying in $\mathbb{R}^{3}$.

By simple extension, we define the projection of any shape $S$ on the manifold $\mathcal{M}$ by $P_{\mathcal{M}}(s)=\tilde{\Phi}_{\mid \mathcal{M}}^{-1}(\tilde{\Phi}(s))$. Note that we do not try to estimate the manifold outside of its limits, as the ones defined by the convex hull of the trainng points in the reduced space. As a consequence, projection of a point located outside the manifold will belong to the border of the manifold.

\subsection{Manifold Denoising}

In the previous section, we estimate the manifold $\mathcal{M}$ by interpolating between training shape samples (i.e. by minimization of an energy functional) subject to constant embedding constraints (Eq. 4). Thus, the manifold $\mathcal{M}$ is assumed to go through every training sample. Unfortunately, this implies that our manifold reconstruction is sensitive to outliers that are mapped among other training samples into the reduced space through the embedding $\tilde{\Phi}$ (Fig 2-a). To alleviate this problem, we propose to use the mapping $\tilde{\Phi}$ and the Euclidean nature of the reduced space to design a denoising functional $E^{\text {denoising }}$. 

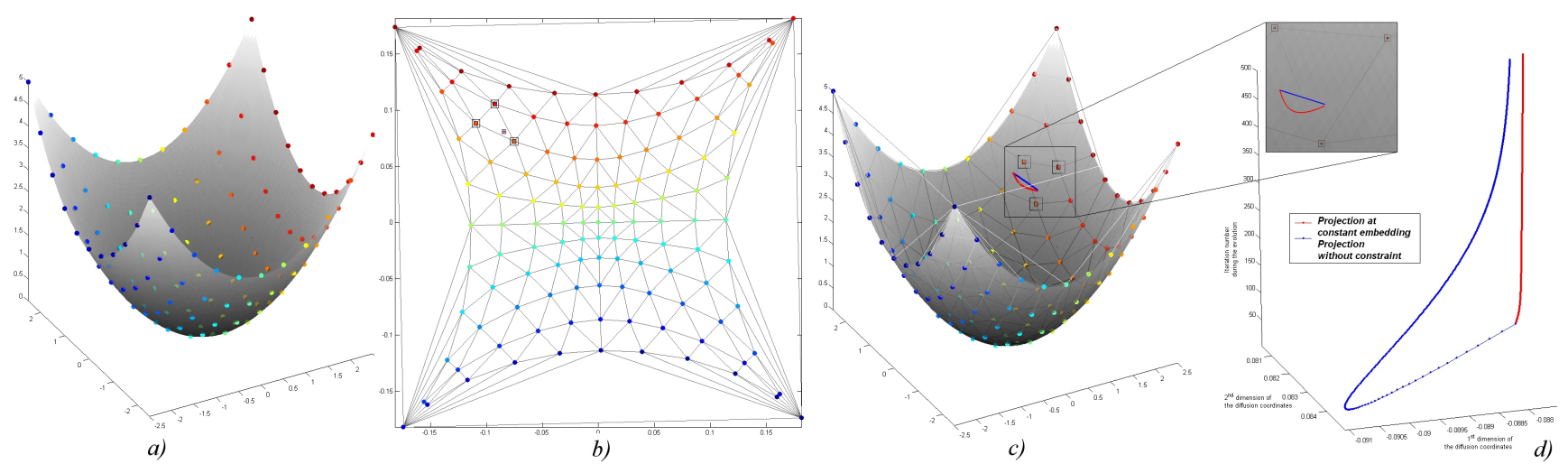

Figure 1. a) Set of point samples lying on the surface given by the equation $f(x, y)=x^{2}+y^{2}$. b) The reduced space and the Delaunay triangulation. c) Projection towards the weighted mean (in blue) and at constant embedding (in red). The Delaunay triangulation is represented in the original space. d) Values of the embedding during the two evolutions

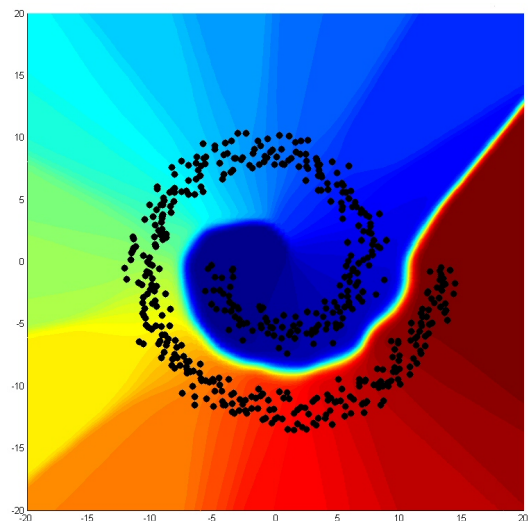

a)

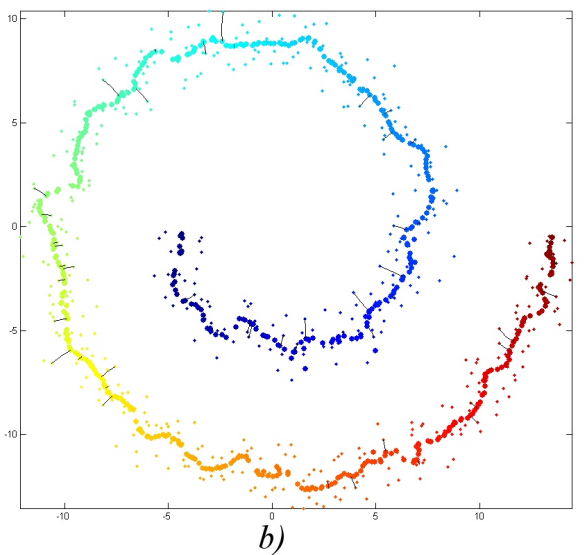

b)

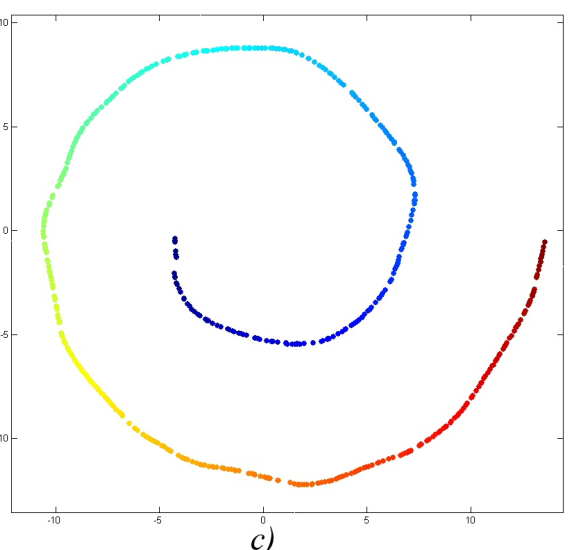

c)

Figure 2. a) The set of point sample with the iso-level set of the embedding. b) After 5 iterations of denoising. Smaller points are orignal data, bigger are denoised data. The black lines are the paths of some points during the evolution c) Final result.

The embedding $\tilde{\Phi}$ captures the intrinsic geometry of the manifold $\mathcal{M}$ by mapping training samples into $\mathbb{R}^{m}$ isometrically with respect to a diffusion distance in the original shape space. It is useful to interpret the mapping as a smoothing filter that absorbs the "noise components orthogonal to the manifold" and maps outliers among valid training samples. In light of this, we propose to use the connectedness of the Delaunay triangulation $\mathbb{D}_{\mathcal{M}}$ in the reduced space to infer connectedness of the training samples in the original space $\mathbb{S}$. For each training sample $s_{i} \in \Gamma$, we identify its set $N_{i}$ of adjacent neighbors that are connected in the Delaunay triangulation $\mathbb{D}_{\mathcal{M}}$. We then define the denoising functional over all training samples:

$$
E^{\text {denoising }}(\Gamma)=\sum_{s_{i} \in \Gamma} \sum_{s_{i, k} \in \mathcal{N}_{i}} d^{2}\left(s_{i}, s_{i, k}\right),
$$

The functional $E^{\text {denoising }}$ is minimized by gradient descent with the additional constraints of preserving the embedding. To do so, we enforce the additional constraint $\forall S_{i} \in \Gamma \tilde{\Phi}\left(S_{i}\right)=$ constant, which can be expressed by $m \times p$ orthogonality conditions in the tangent space (see next section for more detail).

Minimization of the functional $E^{\text {denoising }}$ implements the well-known umbrella-operator, which is a linear approximation of the Laplacian operator [10]. As such, our denoising framework acts as a diffusion process, attracting every shape sample towards the mean shape of its neighbors. In spirit, it is similar to the approach proposed by Hein and Maier in [13]. Yet, it is different in two essential aspects. First, the diffusion process is based on the diffusion distance, which is more robust to outliers than geodesic distance. The connectivity of the manifold $\mathcal{M}$ is directly derived from the Delaunay triangulation $\mathbb{D}_{\mathcal{M}}$. Also, during the evolution, we avoid the time consuming procedure which consists of updating the whole connectivity graph, since we enforce the embedding to remain the same. The works in [18] have some common points in spirit but the method cannot be easily applied to the shape manifold. Finally, as noted in $[10,13]$, there exists a tradeoff between reducing the noise and smoothing the manifold. Minimization of the 
energy Eq. 5 leads to a global flow which smooths the manifold via mean curvature.

\subsection{Shape prior Term}

After denoising the manifold $\mathcal{M}$, we tackle the formulation of the shape prior term. We denote by $E_{\mathcal{N}, \Theta}^{s p}$ the following functional

$$
s \mapsto E_{\mathcal{N}, \Theta}^{s p}(s)=\sum_{s_{i} \in \mathcal{N}} \theta_{i} d^{2}\left(s, s_{i}\right),
$$

where the coefficients $\Theta=\left\{\theta_{0}, \ldots, \theta_{m}\right\}$ are solution to Eq. 4 with $x=\tilde{\Phi}(s)$. We consider the active contour evolution $s: \tau \in \mathbb{R}^{+} \mapsto s(\tau) \in \mathbb{S}$ such that

$$
s(0)=S_{0}, \frac{d s}{d \tau}=-\nabla E_{\mathcal{N}, \Theta}^{s p}(s) .
$$

Minimization of $E_{\mathcal{N}, \Theta}^{s p}$ by gradient flow produces an evolution which attracts the active-contour shape $s$ towards its projection onto the manifold $P_{\mathcal{M}}\left(s_{0}\right)$. Yet, the embedding coordinates $\tilde{\Phi}(s(\tau))$ of the evolving shape $s(\tau)$ are not guaranteed to remain constant during the evolution.

To alleviate this problem, we define the shape prior term $\vec{v}_{s p}$ as the projection of the velocity field $\vec{v}=-\nabla E_{\mathcal{N}, \Theta}^{s p}$ onto the tangent space of $\mathbb{S}_{\tilde{\Phi}(s)}$ at $s$, denoted $\mathbb{T}_{\tilde{\Phi}(s)}$. Using Eq. 1 and Eq. $3, \mathbb{T}_{\tilde{\Phi}(s)}$ can be expressed by $m$ simple orthogonality conditions in the tangent space $\mathbb{T}_{\mathbb{S}}(s)$ of $\mathbb{S}$ at $s$ :

$$
\mathbb{T}_{\tilde{\Phi}(S)}=\left\{\begin{array}{l}
\vec{v} \in \mathbb{T}_{\mathbb{S}}(s) \text { such that } \forall k=1, \ldots, m \\
\sum_{y \in \Gamma}\left\langle\nabla_{s} p(s, y) \mid \vec{v}\right\rangle_{\mathbb{L}^{2}} \Psi_{k}(y)=0
\end{array}\right\},
$$

where $\langle. \mid .\rangle_{\mathbb{L}^{2}}$ corresponds to the $\mathbb{L}^{2}$-dot product in the tangent shape space $\mathbb{T}_{\mathbb{S}}(s)$. Projection of the velocity field $-\nabla E_{\mathcal{N}, \Theta}^{s p}$ onto $\mathbb{T}_{\tilde{\Phi}(s)}$ can then be achieved using the orthogonalization Gram-Schmidt process. This is illustrated in figure 1 and in section 4.

Finally, the general deformable model framework corresponds to solving the following evolution problem:

$$
s(0)=s_{0}, \frac{d s}{d \tau}=-\nabla E^{a c}(s)+\alpha \vec{v}_{s p},
$$

where $\alpha$ is a weighting parameter. Note that at each step of the evolution, we have to align the shape with the training samples using the principal moments before computing its embedding and deriving the shape prior term $\vec{v}_{s p}$.

\section{Applications and Discussion}

\subsection{Segmentation of 2D Cars}

In this example, we illustraste the shape prior term in segmentation tasks of 2D car shapes. We are aiming at segmenting partly occluded cars. In this experiment, the nonlinear prior is the manifold of the $2 \mathrm{~d}$ shapes observed while

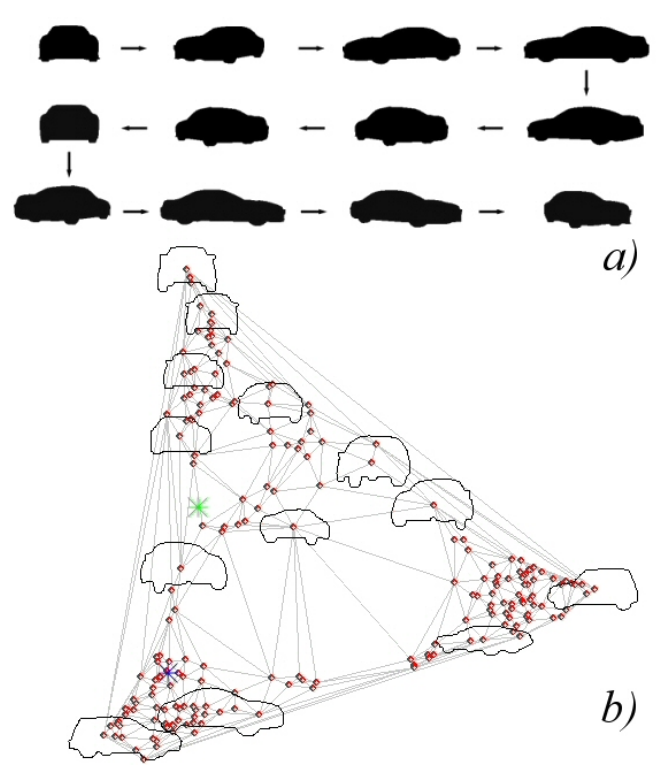

Figure 3. a) 12 shapes for one of the 17 cars used in the dataset. b) Reduced space of the car data set and its Delaunay triangulation.

turning around different cars. The used dataset is made up of 17 cars whose shapes are quite different : Audi A3, Audi TT, BMW Z4, Citroën C3, Chrysler Sebring, Honda Civic, Renault Clio, Delorean DMC-12, Ford Mustang Coupe, Lincoln MKZ, Mercedes S-Class, Lada Oka, Fiat Palio, Nissan 200sx , Nissan Primera, Hyundai Santa Fe and Subaru Forester. For each car, we extracted 12 shapes from the projection of the $3 \mathrm{~d}$ CAD model (fig. 3 a) forming a dataset of 204 shape samples. The shapes are finally stored in the form of distance functions by means of $160 \times 120$ images In the learning stage, the embedding of the car shape manifold is estimated using Diffusion Maps over the dataset. In figure $3 \mathrm{~b}$, we represented the two first dimensions of the diffusion coordinates, which constitutes the reduced space, and the corresponding Delaunay triangulation. Note that the car shapes have a coherent spatial organization in the reduced space.

Without loss of generality, we implemented our surface deformation in the level set framework. We used a simple data term designed to attract the curve towards image edges [19], which gives the following evolution equation:

$$
\begin{aligned}
\partial_{\tau} \bar{D}_{S}(x, \tau) & =g\left(\nabla_{x} I(x)\right)\left[\nu+\varepsilon \kappa\left(\bar{D}_{S}(x, \tau)\right]\right. \\
& *\left|\nabla \bar{D}_{S}(x, \tau)\right|-\alpha \vec{v}_{s p} \cdot \bar{D}_{S}(x, \tau)
\end{aligned}
$$

where $\bar{D}_{S}$ is the signed distance function of the evolving contour. $\kappa\left(\bar{D}_{S}(x)\right)=\operatorname{div}\left(\frac{\nabla_{x} \bar{D}_{(x)}}{\left\|\nabla_{x} \bar{D}_{(}(x)\right\|}\right), I(x)$ and $\nu$ are respectively the mean curvature, the image intensity at location $x$ and a constant speed term to push or pull the contour. $g(\vec{z})=\frac{1}{1+\|\vec{z}\|^{2}}$ is a stopping function for edge extraction. 
In order to demonstrate the influence of our shape prior, we achieved segmentation of partly occluded cars which are not in the initial data set. We also chose images whose the point of views are completely different. We initialized the contour with an ellipse around the car to segment and observed the evolution in both cases, with and without our shape prior. The final results are presented in figure 4.1. Without the shape prior, the energy is obviously minimized on the image edges. However, when the shape prior is incorporated, the new energy overcomes local minima of the data term energy and finally gives the good segmentation.

\subsection{D shapes : Ventricules in medical imaging}

We now use a dataset of 39 ventricules nuclei from Magnetic Resonance Image (MRI). The shapes are aligned using their principal moment before computing their diffusion coordinates. In this experiment, we compare the projection at constant embedding, the neighbors in the Delaunay triangulation of the reduced space and the mean shape obtain from these neighbors. Our deformation surface is again implemented in the level set framework: the distance functions of the ventricule shapes are encoded in $140 \times 75 \times 60$ images. To perform the projection, we start from an ellipsoid aligned on the $3 \mathrm{~d}$ shape set. Its embedding is indicated by the black point in figure 4.2. The nearest shapes in the corresponding Delaunay triangle are easily identified in order to compute the mean shape target and the projection at constant embedding. The projection at constant embedding captures details (on the right side of the ventricule) of closest shapes ( $38 \& 22)$ that the mean shape loose due to its smoothing properties.

\section{Conclusion and Future Work}

In this paper, we have introduced a new deformable model framework that integrates general non-linear shape priors using Diffusion maps. We presented a new projection operator onto a manifold based on the Nyström extension and a Delaunay partitioning of the reduced space. We then provided a variational solution for manifold denoising. Finally, we expressed a new energy term designed to attract a shape towards the manifold at given constant embedding. We demonstrated the strength of our approach by applying these ideas in different experiments (fig. 1, 2, 4.1 4.2) either with synthetic or real data, including in segmentation tasks.

We are currently working on new applications that exploit the concepts presented in this paper. We also expect to use more general data since the only requirement to apply our method is a differentiable kernel.

\section{References}

[1] P. Arias, G. Randall, and G. Sapiro. Connecting the out-ofsample and pre-image problems in kernel methods. In IEEE
International Conference on Pattern Recognition, June 2007. 3,4

[2] M. Belkin and P. Niyogi. Laplacian eigenmaps for dimensionality reduction and data representation. Neural Computation, 15(6):1373-1396, 2003. 2

[3] Y. Bengio, J.-F. Paiement, P. Vincent, O. Delalleau, N. Le Roux, and M. Ouimet. Out-of-sample extensions for lle, isomap, mds, eigenmaps, and spectral clustering. In S. Thrun, L. K. Saul, and B. Schölkopf, editors, Advances in Neural Information Processing Systems 16. MIT Press, Cambridge, MA, 2004. 2, 3

[4] A. M. Bronstein, M. M. Bronstein, and R. Kimmel. Generalized multidimensional scaling: a framework for isometryinvariant partial surface matching. Proc. National Academy of Sciences (PNAS), 103(5):1168-1172, January 2006. 2

[5] G. Charpiat, O. Faugeras, and R. Keriven. Approximations of shape metrics and application to shape warping and empirical shape statistics. Foundations of Computational Mathematics, 5(1):1-58, 2005. 1, 3, 4

[6] G. Charpiat, O. Faugeras, R. Keriven, and P. Maurel. Distance-based shape statistics. In IEEE International Conference on Acoustics, Speech and Signal Processing, volume 5, pages 925-928, 2006. 2

[7] R. Coifman, S. Lafon, A. Lee, M. Maggioni, B. Nadler, F. Warner, and S. Zucker. Geometric diffusions as a tool for harmonic analysis and structure definition of data: Diffusion maps. PNAS, 102(21):7426-7431, 2005. 2, 3, 4

[8] T. Cootes, C. Taylor, D. Cooper, and J. Graham. Active shape models-their training and applications. Computer Vision and Image Understanding, 61(1):38-59, 1995. 1

[9] D. Cremers, T. Kohlberger, and C. Schnörr. Nonlinear shape statistics in mumford shah based segmentation. In European Conference on Computer Vision, pages 93-108, 2002. 1

[10] M. Desbrun, M. Meyer, P. Schröder, and A. H. Barr. Implicit fairing of irregular meshes using diffusion and curvature flow. Computer Graphics, 33(Annual Conference Series):317-324, 1999. 5

[11] P. Etyngier, R. Keriven, and J.-P. Pons. Towards segmentation based on a shape prior manifold. In 1st International Conference on Scale Space and Variational Methods in Computer Vision, pages 895-906, Ishia, Italy, May 2007. 1

[12] M. Hein, A. J.-Y., and U. Von Luxburg. Graph laplacians and their convergence on random neighborhood graphs. Journal of Machine Learning Research, 8:1325-1370, 2007. 2, 3

[13] M. Hein and M. Maier. Manifold denoising. In Advances in Neural Information Processing Systems 20, Cambridge, MA, USA, 2006. MIT Press. 5

[14] J. T. Kwok and I. W. Tsang. The pre-image problem in kernel methods. In International Conference on Machine Learning, pages $408-415,2003.4$

[15] S. Lafon, Y. Keller, and R. R. Coifman. Data fusion and multicue data matching by diffusion maps. IEEE Transactions on Pattern Analysis and Machine Intelligence, 28(11):17841797, 2006. 2, 3

[16] S. Lafon and A. B. Lee. Diffusion maps and coarse-graining: a unified framework for dimensionality reduction, graph partitioning, and data set parameterization. IEEE Transactions 

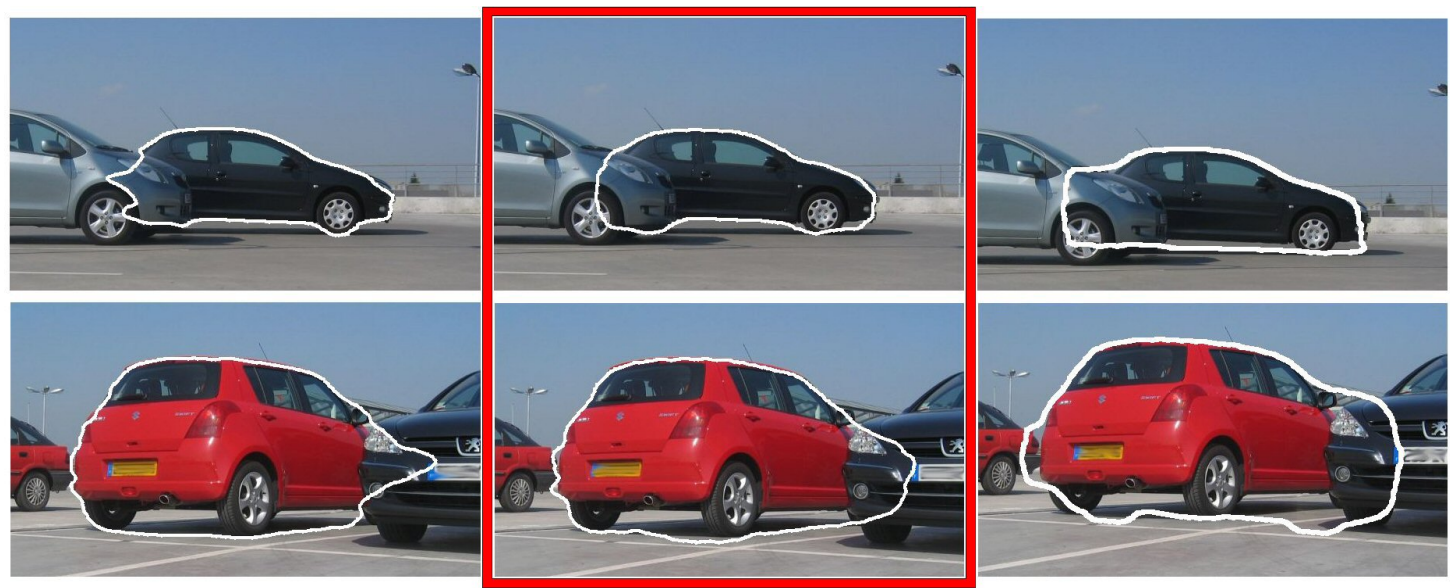

Figure 4. Segmentation of a Peugeot 206 (first row) and a Suzuki Swift (second row). First column: Segmentation with data term only. Second column: segmentation with our shape prior. The embedding of the final shape is denoted by a blue cross and a green cross respectively for the Peugeot 206 and the Suzuki Swift in figure 3 b) Third column: Segmentation with the nearest neighbor in the shape space as prior (such choice is not relevant compared to the nearest neigbors in the diffusion coordinates

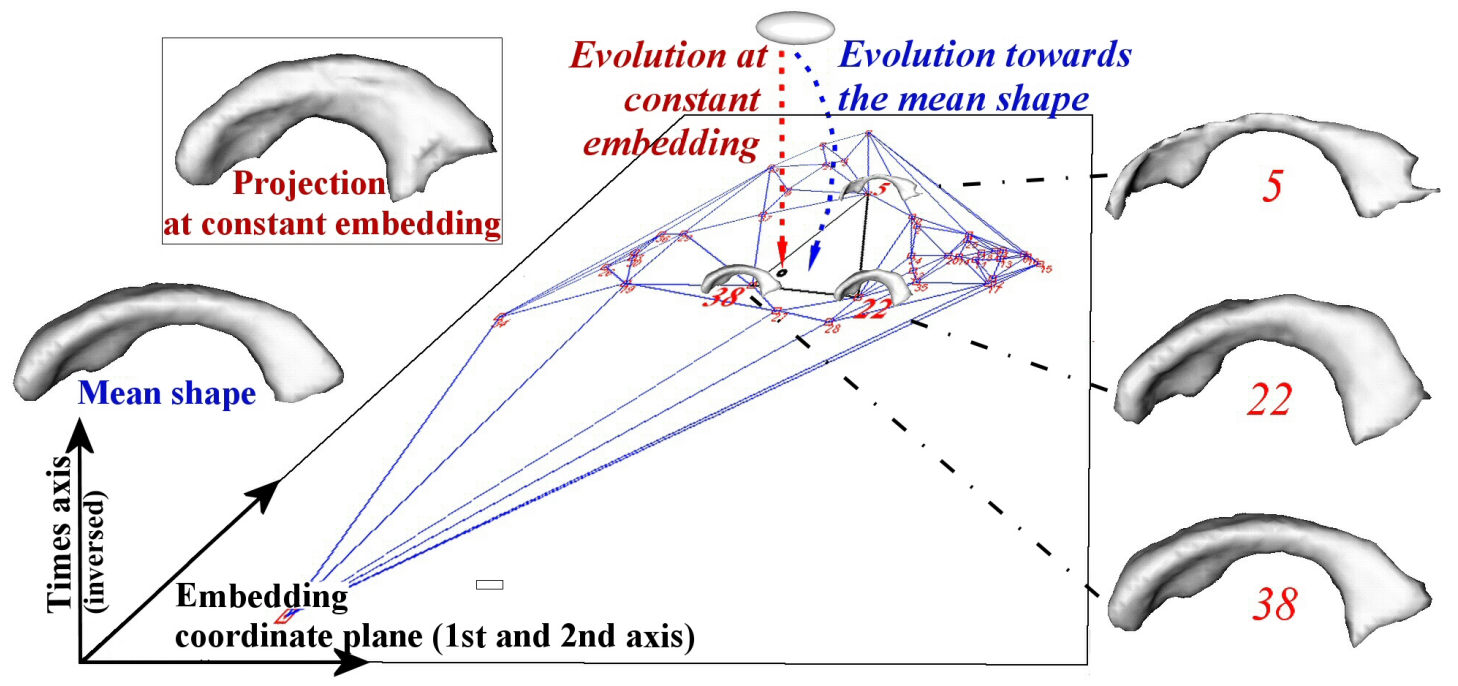

Figure 5. The ventricule manifold: Comparison of the evolution towards the mean shape and the evolution at constant embedding

on Pattern Analysis and Machine Intelligence, 28(9):13931403, 2006. 2, 3

[17] M. Leventon, E. Grimson, and O. Faugeras. Statistical shape influence in geodesic active contours. In IEEE Conference on Computer Vision and Pattern Recognition, pages 316-323, 2000. 1

[18] D. Levin. Geometrical Modeling for Scientific Visualization. Brunnett, G.; Hamann, B.; Mller, H.; Linsen, L. Eds, chapter Mesh-Independent Surface Interpolation. Springer, 2004. 5

[19] S. Osher and R. Fedkiw. Level set methods: an overview and some recent results. Journal of Computational Physics, 169(2):463-502, 2001. 6

[20] S. Osher and J. Sethian. Fronts propagating with curvature- dependent speed: Algorithms based on Hamilton-Jacobi formulations. Journal of Comp. Physics, 79(1):12-49, 1988. 1

[21] M. Rousson and N. Paragios. Shape priors for level set representations. In European Conference on Computer Vision, volume 2, pages 78-92, 2002. 1

[22] S. Roweis and L. Saul. Nonlinear dimensionality reduction by locally linear embedding. Science, 290:2323-2326, 2000. 2

[23] A. Tsai, A. Yezzi, W. Wells, C. Tempany, D. Tucker, A. Fan, W. Grimson, and A. Willsky. A shape-based approach to the segmentation of medical imagery using level sets. IEEE Transactions on Medical Imaging, 22(2):137-154, 2003. 1 